\title{
THE HOWE DUALITY CONJECTURE: QUATERNIONIC CASE
}

\author{
WEE TECK GAN AND BINYONG SUN \\ In celebration of \\ Professor Roger Howe's 70th birthday
}

\begin{abstract}
We complete the proof of the Howe duality conjecture in the theory of local theta correspondence by treating the remaining case of quaternionic dual pairs in arbitrary residual characteristic.
\end{abstract}

\section{Introduction}

Let $\mathrm{F}$ be a non-archimedean local field of characteristic not 2. Let $W$ be a finite-dimensional symplectic vector space over $\mathrm{F}$ with symplectic form $\langle,\rangle_{W}$. Write

$$
1 \rightarrow\left\{1, \varepsilon_{W}\right\} \rightarrow \widetilde{\mathrm{Sp}}(W) \rightarrow \mathrm{Sp}(W) \rightarrow 1
$$

for the metaplectic double cover of the symplectic group $\operatorname{Sp}(W)$. It does not split unless $W=0$. Denote by $\mathrm{H}(W):=W \times \mathrm{F}$ the Heisenberg group attached to $W$, with group multiplication

$$
(u, \alpha)(v, \beta):=\left(u+v, \alpha+\beta+\langle u, v\rangle_{W}\right), \quad u, v \in W, \alpha, \beta \in \mathrm{F} .
$$

Then $\widetilde{\operatorname{Sp}}(W)$ acts on $\mathrm{H}(W)$ as group automorphisms through the action of $\operatorname{Sp}(W)$ on $W$, and we may form the semi-direct product $\widetilde{\mathrm{J}}(W):=\widetilde{\mathrm{Sp}}(W) \ltimes \mathrm{H}(W)$.

Fix an arbitrary non-trivial unitary character $\psi: \mathrm{F} \rightarrow \mathbb{C}^{\times}$. Up to isomorphism, there is a unique smooth representation $\omega_{\psi}$ of $\widetilde{\mathrm{J}}(W)$ (called a Weil representation) such that (cf. [We, Section IV.43])

- $\left.\omega_{\psi}\right|_{\mathrm{H}(W)}$ is irreducible and has central character $\psi$;

- $\varepsilon_{W} \in \widetilde{\mathrm{Sp}}(W)$ acts through the scalar multiplication by -1 .

Unless $W=0$, the above second condition is a consequence of the first one.

Denote by $\tau$ the involution of $\operatorname{End}_{F}(W)$ specified by

$$
\langle x \cdot u, v\rangle_{W}=\left\langle u, x^{\tau} \cdot v\right\rangle_{W}, \quad u, v \in W, x \in \operatorname{End}_{\mathrm{F}}(W) .
$$

Let $\left(A, A^{\prime}\right)$ be a pair of $\tau$-stable semisimple $\mathrm{F}$-subalgebras of $\operatorname{End}_{\mathrm{F}}(W)$ which are mutual centralizers of each other. Put $G:=A \cap \operatorname{Sp}(W)$ and $G^{\prime}:=A^{\prime} \cap \operatorname{Sp}(W)$, which are closed subgroups of $\operatorname{Sp}(W)$. Following Howe, we call the pair $\left(G, G^{\prime}\right)$ so obtained a reductive dual pair in $\operatorname{Sp}(W)$. We say that the pair $\left(A, A^{\prime}\right)$ (or the reductive dual pair $\left(G, G^{\prime}\right)$ ) is irreducible of type I if $A$ (or equivalently $A^{\prime}$ ) is a simple algebra, and say that it is irreducible of type II if $A$ (or equivalently $A^{\prime}$ ) is the product of two simple algebras which are exchanged by $\tau$. A complete classification of such dual pairs has been given by Howe.

Date: July 17, 2015.

2000 Mathematics Subject Classification. Primary 11F27, Secondary 22E50.

Key words and phrases. Howe duality conjecture, theta correspondence, quaternionic dual pair. 
For every closed subgroup $H$ of $\operatorname{Sp}(W)$, write $\widetilde{H}$ for the double cover of $H$ induces by the metaplectic cover (1.1). Then $\widetilde{G}$ and $\widetilde{G}^{\prime}$ commute with each other inside the group $\widetilde{\operatorname{Sp}}(W)$ (cf. MVW, Chapter 2, Lemma II.5]). Thus, the Weil representation $\omega_{\psi}$ can be regarded as a representation of $\widetilde{G} \times \widetilde{G}^{\prime}$.

For every $\pi \in \operatorname{Irr}(\widetilde{G})$, put

$$
\Theta_{\psi}(\pi):=\left(\omega_{\psi} \otimes \pi^{\vee}\right)_{\widetilde{G}},
$$

to be viewed as a smooth representation of $\widetilde{G}^{\prime}$. Here and as usual, a superscript " $\vee$ " indicates the contragredient representation, a subscript group indicates the coinvariant space, and "Irr" indicates the set of isomorphism classes of irreducible admissible representations of the group. It was proved by Kudla $\left[\mathbf{K}\right.$ that the representation $\Theta_{\psi}(\pi)$ is admissible and has finite length. Denote by $\theta_{\psi}(\pi)$ the maximal semisimple quotient of $\Theta_{\psi}(\pi)$, which is called the theta lift of $\pi$. In this paper, we complete the proof of the following Howe duality conjecture.

\section{The Howe Duality Conjecture}

For every reductive dual pair $\left(G, G^{\prime}\right)$ and every $\pi \in \operatorname{Irr}(\widetilde{G})$, the theta lift $\theta_{\psi}(\pi)$ is irreducible if it is non-zero.

The Howe duality conjecture is easily reduced to the case when the pair $\left(A, A^{\prime}\right)$ is irreducible (of type I or II). It has been proved by Waldspurger [Wa when the residual characteristic of $\mathrm{F}$ is not 2 . For irreducible reductive dual pairs of type II, the conjecture was proved in full and more simply by Minguez in [M]. Every irreducible reductive dual pair of type I is an orthogonal-symplectic dual pair, a unitary dual pair, or a quaternionic dual pair [H1, Section 5]. For orthogonal-symplectic dual pairs and unitary dual pairs, the conjecture was proved in GT. (it was earlier proved in LSTi] that $\theta_{\psi}(\pi)$ is multiplicity free). For the remaining case of quaternionic dual pairs, only a partial result was obtained in GT (for Hermitian representations). The reason is that GT makes use of the MVW-involution on the category of smooth representations, and it has been shown in [LSTa] that such an involution does not exist in the quaternionic case.

The purpose of this paper is to explain how the use of the MVW-involution can be avoided, thus completing the proof of the Howe duality conjecture in the quaternionic case. The lack of an MVWinvolution necessitates relating the theta lifts of $\pi$ and $\pi^{\vee}$, and the key new ingredient is provided by the following consequence of the conservation relations shown in [SZ, Equalities (12)].

Lemma 1.1. Assume that $\left(G, G^{\prime}\right)$ is irreducible. Then for every $\pi \in \operatorname{Irr}(\widetilde{G})$,

$$
\theta_{\psi}(\pi) \neq 0 \quad \text { if and only if } \quad \theta_{\bar{\psi}}\left(\pi^{\vee}\right) \neq 0,
$$

where $\bar{\psi}$ denotes the complex conjugation of $\psi$.

In proving the Howe duality conjecture, one needs to strengthen Lemma 1.1 to the identity

$$
\left(\theta_{\psi}(\pi)\right)^{\vee} \cong \theta_{\bar{\psi}}\left(\pi^{\vee}\right) \quad \text { for every } \pi \in \operatorname{Irr}(\widetilde{G}) .
$$

Hence, the main result of this paper is the following theorem, which encompasses the Howe duality conjecture and the identity (1.3).

Theorem 1.2. Assume that $\left(G, G^{\prime}\right)$ is irreducible, and the size of $G$ is no smaller than that of $G^{\prime}$. Then for all $\pi, \sigma \in \operatorname{Irr}(\widetilde{G})$,

- $\theta_{\psi}(\pi)$ is irreducible if it is non-zero;

- if $\theta_{\psi}(\pi) \cong \theta_{\psi}(\sigma) \neq 0$, then $\pi \cong \sigma$;

- $\left(\theta_{\psi}(\pi)\right)^{\vee} \cong \theta_{\bar{\psi}}\left(\pi^{\vee}\right)$.

Consequently, the Howe duality conjecture holds for both $\left(G, G^{\prime}\right)$ and $\left(G^{\prime}, G\right)$, and for every $\pi^{\prime} \in$ $\operatorname{Irr}\left(\widetilde{G}^{\prime}\right),\left(\theta_{\psi}\left(\pi^{\prime}\right)\right)^{\vee} \cong \theta_{\bar{\psi}}\left(\pi^{\prime \vee}\right)$. 
Here the size of $G$ is defined to be

$\operatorname{size}(G):= \begin{cases}\frac{n_{A}}{2}+\frac{\operatorname{dim}_{K} A^{\tau=-1}}{n_{A}}, & \text { if }\left(G, G^{\prime}\right) \text { is an orthogonal-symplectic or quaternionic dual pair; } \\ n_{A}, & \text { otherwise }\end{cases}$

where $K$ denotes the center of $A, A^{\tau=-1}:=\left\{\alpha \in A \mid \alpha^{\tau}=-\alpha\right\}$, and $n_{A}$ denotes the integer such that $\operatorname{rank}_{K} A=n_{A}^{2}$. The size of $G^{\prime}$ is analogously defined.

In fact, exploiting Lemma 1.1, Theorem 1.2 is equivalent to the following proposition.

Proposition 1.3. Assume that $\left(G, G^{\prime}\right)$ is irreducible, and the size of $G$ is no smaller than that of $G^{\prime}$. Then for all $\pi, \sigma \in \operatorname{Irr}(\widetilde{G})$,

$$
\operatorname{dim} \operatorname{Hom}_{\widetilde{G}^{\prime}}\left(\theta_{\psi}(\pi) \otimes \theta_{\bar{\psi}}(\sigma), \mathbb{C}\right) \leq \operatorname{dim} \operatorname{Hom}_{\widetilde{G}}(\pi \otimes \sigma, \mathbb{C}) .
$$

In what follows, we show that Lemma 1.1 and Proposition 1.3 imply Theorem 1.2

Proof of (Lemma 1.1 + Proposition $1.3 \Longrightarrow$ Theorem 1.2)

For every $\pi \in \operatorname{Irr}(\widetilde{G})$ and $\pi^{\prime} \in \operatorname{Irr}\left(\widetilde{G}^{\prime}\right)$, write

$$
\mathrm{m}_{\psi}\left(\pi, \pi^{\prime}\right):=\operatorname{dim} \operatorname{Hom}_{\widetilde{G} \times \widetilde{G}^{\prime}}\left(\omega_{\psi}, \pi \otimes \pi^{\prime}\right)
$$

and define $\mathrm{m}_{\bar{\psi}}\left(\pi, \pi^{\prime}\right)$ similarly. We claim that

$$
\mathrm{m}_{\psi}\left(\pi, \pi^{\prime}\right) \neq 0 \text { if and only if } \mathrm{m}_{\bar{\psi}}\left(\pi^{\vee}, \pi^{\prime \vee}\right) \neq 0 \text {. }
$$

It is easy to see that (1.4) and (1.5) imply Theorem 1.2

To prove the claim, we first assume that $\mathrm{m}_{\psi}\left(\pi, \pi^{\prime}\right) \neq 0$. Applying Lemma 1.1 to the pair $\left(G^{\prime}, G\right)$, we see that $\mathrm{m}_{\bar{\psi}}\left(\sigma, \pi^{\prime \vee}\right) \neq 0$ for some $\sigma \in \operatorname{Irr}(\widetilde{G})$. The inequality (1.4) then implies that $\sigma \cong \pi^{\vee}$ and hence $\mathrm{m}_{\bar{\psi}}\left(\pi^{\vee}, \pi^{\prime \vee}\right) \neq 0$. Similarly, if $\mathrm{m}_{\bar{\psi}}\left(\pi^{\vee}, \sigma^{\vee}\right) \neq 0$ then $\mathrm{m}_{\psi}(\pi, \sigma) \neq 0$. This proves the claim (1.5), and therefore shows that Lemma 1.1 and Proposition 1.3 imply Theorem 1.2

In view of the above, the main body of our paper will be devoted to the proof of Proposition 1.3 .

Remarks: (a) Reductive dual pairs as defined in this paper include the following case: $G$ is the quaternionic orthogonal group attached to a one-dimensional quaternionic skew Hermitian space, and $G^{\prime}$ is the quaternionic symplectic group attached to a non-zero quaternionic Hermitian space (see the next section). In this case, $G^{\prime}$ is strictly contained in the centralizer of $G$ in the symplectic group.

(b) Although in the statements of [Ya, Theorems 1.3 and 1.4] (see Lemma 2.2) and [SZ, Equalities (12)], the authors assume that the base field F has characteristic zero, their methods prove the same results for all non-archimedean local field $\mathrm{F}$ of characteristic not 2.

(c) For type II irreducible reductive dual pairs, the identity (1.3) is a consequence of [M], Theorem 1], in which the explicit theta lifts are determined in terms of the Langlands parameters. For orthogonalsymplectic and unitary dual pairs, (1.3) is a consequence of the MVW involution ( $c f$. S, Theorem $1.4])$.

\section{Acknowledgements}

This paper is essentially completed during the conference in honor of Professor Roger Howe on the occasion of his 70th birthday. We thank the organisers of the conference (James Cogdell, Ju-Lee Kim, Jian-Shu Li, David Manderscheid, Gregory Margulis, Cheng-Bo Zhu and Gregg Zuckerman) for their kind invitation to speak at the conference and for providing local support.

During the Howe conference, the first author presented his paper [GT] with S. Takeda on the proof of the Howe duality conjecture for orthogonal-symplectic and unitary dual pairs and mentioned 
that the quaternionic case still needed to be addressed because of the lack of the MVW involution. He expressed the hope that some trick could be found by the end of the conference to deal with the quaternionic case. The following day, the second author realised that a consequence of the conservation relation shown in his paper $\mathrm{SZ}$ ] with C.-B. Zhu could serve as a replacement for the MVW-involution: this is the innocuous-looking statement (1.2) above. The two authors were able to verify the details in the next two days, thus completing the proof of the Howe duality conjecture in the quaternionic case. It gives us great pleasure to dedicate this paper to Roger Howe, who had initiated this whole area of research and formulated this conjecture at the beginning of his career. We hope that it gives him much satisfaction in seeing this conjecture completely resolved at the time of his retirement from Yale.

W.T. Gan is partially supported by an MOE Tier Two grant R-146-000-175-112. B. Sun is supported in part by the NSFC Grants 11222101 and 11321101.

\section{The doubling method}

We will only treat the quaternionic case in the proof of Proposition 1.3 , since it is previously known in all other cases. Let $\mathrm{F}$ be a nonarchimedean local field of characteristic not 2 , with $|\cdot|_{\mathrm{F}}$ denoting the normalized absolute value on $\mathrm{F}$. Let $\mathrm{D}$ be a central division quaternion algebra over $\mathrm{F}$, which is unique up to isomorphism. Denote by $\iota: D \rightarrow D$ the quaternion conjugation of $\mathrm{D}$. We consider an $\epsilon$-Hermitian right $\mathrm{D}$-vector space $U$, and an $\epsilon^{\prime}$-Hermitian left D-vector space $V$, where $\epsilon= \pm 1$ and $\epsilon^{\prime}=-\epsilon$. To be precise, $U$ is a finite dimensional right D-vector space, equipped with a non-degenerate F-bilinear map

$$
\langle,\rangle_{U}: U \times U \rightarrow \mathrm{D}
$$

satisfying

$$
\left\langle u, u^{\prime} \alpha\right\rangle_{U}=\left\langle u, u^{\prime}\right\rangle_{U} \alpha \quad \text { and } \quad\left\langle u, u^{\prime}\right\rangle_{U}=\epsilon\left\langle u^{\prime}, u\right\rangle_{U}^{\iota}, \quad u, u^{\prime} \in U, \alpha \in \mathrm{D} .
$$

Similarly, $V$ is a finite dimensional left D-vector space and is equipped with a form $\langle,\rangle_{V}: V \times V \rightarrow \mathrm{D}$ with the analogous properties. The tensor product $W:=U \otimes_{\mathrm{D}} V$ is a symplectic space over $\mathrm{F}$ under the bilinear form

$$
\left\langle u \otimes v, u^{\prime} \otimes v^{\prime}\right\rangle_{W}:=\frac{\left\langle u, u^{\prime}\right\rangle_{U}\left\langle v, v^{\prime}\right\rangle_{V}^{\iota}+\left\langle v, v^{\prime}\right\rangle_{V}\left\langle u, u^{\prime}\right\rangle_{U}^{\iota}}{2}, \quad u, u^{\prime} \in U, v, v^{\prime} \in V .
$$

Throughout the paper, we fix two quadratic (order at most 2) characters $\chi_{U}, \chi_{V}: \mathrm{F}^{\times} \rightarrow\{ \pm 1\}$ determined by the discriminants of $U$ and $V$ respectively. More precisely, we have:

$$
\chi_{V}(\alpha)=\left((-1)^{\operatorname{dim} V} \prod_{i=1}^{\operatorname{dim} V}\left\langle e_{i}, e_{i}\right\rangle_{V}\left\langle e_{i}, e_{i}\right\rangle_{V}^{\iota}, \alpha\right)_{\mathrm{F}}, \quad \alpha \in \mathrm{F}^{\times}
$$

where $e_{1}, e_{2}, \cdots, e_{\operatorname{dim} V}$ is an orthogonal basis of $V$, and $(,)_{\mathrm{F}}$ denotes the quadratic Hilbert symbol for F. Likewise, one has the analogous definition for $\chi_{U}$. Note that if $\epsilon=1$, then the isometry class of $U$ is determined by its dimension, and $\chi_{U}$ only depends on the parity of $\operatorname{dim} U$; likewise, if $\epsilon^{\prime}=1$, $\chi_{V}$ only depends on the parity of $\operatorname{dim} V$.

Denote by $W^{-}$the space $W$ equipped with the form scaled by -1 . Write $W^{\square}:=W \oplus W^{-}$for the orthogonal direct sum, which contains $W^{\triangle}:=\left\{(u, u) \in W^{\square} \mid u \in W\right\}$ as a Lagrangian subspace. Define $U^{-}, V^{-}, U^{\square}, V^{\square}, U^{\triangle}, V^{\triangle}$ similarly. Then we have obvious identifications of symplectic spaces

$$
W^{-}=U^{-} \otimes_{\mathrm{D}} V=U \otimes_{\mathrm{D}} V^{-} \quad \text { and } \quad W^{\square}=U^{\square} \otimes_{\mathrm{D}} V=U \otimes_{\mathrm{D}} V^{\square} .
$$

Let $\mathrm{G}(U)$ denote the isometry group of $U$, and similarly for other groups. Then we have identifications

$$
\mathrm{G}(U)=\mathrm{G}\left(U^{-}\right) \quad \text { and } \quad \mathrm{G}(V)=\mathrm{G}\left(V^{-}\right)
$$


and inclusions

$$
\mathrm{G}(U) \times \mathrm{G}\left(U^{-}\right) \subset \mathrm{G}\left(U^{\square}\right) \quad \text { and } \quad \mathrm{G}(V) \times \mathrm{G}\left(V^{-}\right) \subset \mathrm{G}\left(V^{\square}\right) .
$$

Denote by $\mathrm{P}\left(U^{\triangle}\right)$ the parabolic subgroup of $\mathrm{G}\left(U^{\square}\right)$ stabilizing $U^{\triangle}$. Likewise, denote by $\mathrm{P}\left(V^{\triangle}\right)$ the parabolic subgroup of $\mathrm{G}\left(V^{\square}\right)$ stabilizing $V^{\triangle}$.

Let $\omega$ and $\omega^{-}$be irreducible admissible smooth representations of $\mathrm{H}(W)$ and $\mathrm{H}\left(W^{-}\right)$, respectively, both with central character $\psi$. Then the representation $\omega^{\square}:=\omega \otimes \omega^{-}$of $\mathrm{H}(W) \times \mathrm{H}\left(W^{-}\right)$descends to a representation of $\mathrm{H}\left(W^{\square}\right)$ through the surjective homomorphism

$$
\mathrm{H}(W) \times \mathrm{H}\left(W^{-}\right) \rightarrow \mathrm{H}\left(W^{\square}\right), \quad((u, \alpha),(v, \beta)) \mapsto((u, v), \alpha+\beta) .
$$

This representation of $\mathrm{H}\left(W^{\square}\right)$ uniquely extends to the group $\mathrm{G}\left(U^{\square}\right) \ltimes \mathrm{H}\left(W^{\square}\right)$ such that (cf. SW. Theorem 4.7])

$$
\lambda_{\triangle}(g \cdot \phi)=\chi_{V}\left(\operatorname{det}\left(\left.g\right|_{U^{\Delta}}\right)\right)\left|\operatorname{det}\left(\left.g\right|_{U^{\triangle}}\right)\right|_{\mathrm{F}}^{\operatorname{dim} V} \lambda_{\triangle}(\phi), \quad \phi \in \omega^{\square}, g \in \mathrm{P}\left(U^{\triangle}\right),
$$

where $\lambda_{\triangle}$ denotes the unique (up to scalar multiplication) non-zero $W^{\triangle}$-invariant linear functional on $\omega^{\square}$ and det denotes the reduced norm. Similarly, this representation of $\mathrm{H}\left(W^{\square}\right)$ uniquely extends to the group $\mathrm{G}\left(V^{\square}\right) \ltimes \mathrm{H}\left(W^{\square}\right)$ such that

$$
\lambda_{\triangle}\left(g^{\prime} \cdot \phi\right)=\chi_{U}\left(\operatorname{det}\left(\left.g^{\prime}\right|_{V} \Delta\right)\right)\left|\operatorname{det}\left(\left.g^{\prime}\right|_{V}\right)\right|_{\mathrm{F}}^{\operatorname{dim} U} \lambda_{\triangle}(\phi), \quad \phi \in \omega^{\square}, \quad g^{\prime} \in \mathrm{P}\left(V^{\triangle}\right) .
$$

We extend the representation $\omega$ to $(\mathrm{G}(U) \times \mathrm{G}(V)) \ltimes \mathrm{H}(W)$ and extend the representation $\omega^{-}$to $\left(\mathrm{G}\left(U^{-}\right) \times \mathrm{G}\left(V^{-}\right)\right) \ltimes \mathrm{H}\left(W^{-}\right)$such that

$$
\left(\left(g, g^{\prime}\right) \cdot \phi\right) \otimes\left(\left(h, h^{\prime}\right) \cdot \phi^{-}\right)=g h \cdot\left(g^{\prime} h^{\prime} \cdot\left(\phi \otimes \phi^{-}\right)\right)=g^{\prime} h^{\prime} \cdot\left(g h \cdot\left(\phi \otimes \phi^{-}\right)\right),
$$

for all $(g, h) \in \mathrm{G}(U) \times \mathrm{G}\left(U^{-}\right),\left(g^{\prime}, h^{\prime}\right) \in \mathrm{G}(V) \times \mathrm{G}\left(V^{-}\right), \phi \in \omega$ and $\phi^{-} \in \omega^{-}$. Then $\omega$ and $\omega^{-}$are contragredient to each other with respect to the isomorphism

$$
(\mathrm{G}(U) \times \mathrm{G}(V)) \ltimes \mathrm{H}(W) \rightarrow\left(\mathrm{G}\left(U^{-}\right) \times \mathrm{G}\left(V^{-}\right)\right) \ltimes \mathrm{H}\left(W^{-}\right), \quad\left(\left(g, g^{\prime}\right),(u, \alpha)\right) \mapsto\left(\left(g, g^{\prime}\right),(u,-\alpha)\right) .
$$

If necessary, we also write $\omega_{U, V, \psi}$ for the representation $\omega$ of $(\mathrm{G}(U) \times \mathrm{G}(V)) \ltimes \mathrm{H}(W)$, and write $\omega_{U, V, \psi}^{-}$ for the representation $\omega^{-}$of $\left(\mathrm{G}\left(U^{-}\right) \times \mathrm{G}\left(V^{-}\right)\right) \ltimes \mathrm{H}\left(W^{-}\right)$, to emphasize their dependence on $U, V$ and $\psi$.

Thus, we have defined a splitting of (the pushout via $\{ \pm 1\} \hookrightarrow \mathbb{C}^{\times}$of) the metaplectic cover $\widetilde{\mathrm{G}}(U)$ and $\widetilde{\mathrm{G}}(V)$ over $\mathrm{G}(U)$ and $\mathrm{G}(V)$ respectively, so that the Weil representation $\omega_{U, V, \psi}$ is a representation of the linear group $\mathrm{G}(U) \times \mathrm{G}(V)$. Such a splitting is unique over $\mathrm{G}(U)$ if $U$ is quaternionic-Hermitian of dimension $>1$, but is not unique if $U$ is quaternionic-skew-Hermitian (as one can twist by quadratic characters of $\mathrm{G}(U))$. For the purpose of formulating and proving the Howe duality conjecture, there is no loss of generality in working with a fixed splitting.

More precisely, as in the introduction, for every $\pi \in \operatorname{Irr}(\mathrm{G}(U))$, put

$$
\Theta_{\omega}(\pi):=\left(\omega \otimes \pi^{\vee}\right)_{\mathrm{G}(U)},
$$

and define the theta lift $\theta_{\omega}(\pi)$ to be the maximal semisimple quotient of $\Theta_{\omega}(\pi)$. Similarly, the theta lift $\theta_{\omega}\left(\pi^{\prime}\right)$ is defined for all $\pi^{\prime} \in \operatorname{Irr}(\mathrm{G}(V))$. The theta lifts with respect to other oscillator representations, such as $\theta_{\omega^{-}}$, are analogously defined.

Put

$$
s_{U, V}:=\left(\operatorname{dim} U+\frac{\epsilon}{4}\right)-\left(\operatorname{dim} V+\frac{\epsilon^{\prime}}{4}\right) \quad \text { and } \quad s_{V, U}:=\left(\operatorname{dim} V+\frac{\epsilon^{\prime}}{4}\right)-\left(\operatorname{dim} U+\frac{\epsilon}{4}\right)=-s_{U, V} .
$$

The following is a reformulation of Proposition 1.3 in the quaternionic case, using the notations introduced above. 
Proposition 2.1. If $s_{U, V}>0$, then for all $\pi, \sigma \in \operatorname{Irr}(\mathrm{G}(U))$,

$$
\operatorname{dim} \operatorname{Hom}_{\mathrm{G}(V)}\left(\theta_{\omega}(\pi) \otimes \theta_{\omega^{-}}(\sigma), \mathbb{C}\right) \leq \operatorname{dim} \operatorname{Hom}_{\mathrm{G}(U)}(\pi \otimes \sigma, \mathbb{C}) .
$$

The linear functional $\lambda_{\triangle}$ of (2.2) induces a $\mathrm{G}\left(U^{\square}\right)$-intertwining linear map

$$
\omega^{\square} \rightarrow \mathrm{I}\left(s_{V, U}\right), \quad \phi \mapsto\left(g \mapsto \lambda_{\triangle}(g \cdot \phi)\right) .
$$

Here for each $s \in \mathbb{C}$,

$$
\mathrm{I}(s):=\operatorname{Ind}_{\mathrm{P}\left(U^{\triangle}\right)}^{\mathrm{G}\left(U^{\square}\right)}\left(\chi_{V}\left|\operatorname{det}_{U} \Delta\right|_{\mathrm{F}}^{s}\right),
$$

where $\operatorname{det}_{U} \triangle: \operatorname{GL}\left(U^{\triangle}\right) \rightarrow \mathrm{F}^{\times}$denotes the reduced norm map, and $\chi_{V}$ is viewed as a character of $\operatorname{GL}\left(U^{\triangle}\right)$ via the pullback through this map. Throughout this paper, Ind will denote the normalised parabolic induction functor.

Denote by $\mathrm{G}(V)^{\triangle}$ the group $\mathrm{G}(V)$ diagonally embedded in $\mathrm{G}(V) \times \mathrm{G}\left(V^{-}\right)$, to be viewed as a subgroup of $\mathrm{G}\left(V^{\square}\right)$.

Lemma 2.2. The linear map (2.4) induces a $\mathrm{G}\left(U^{\square}\right)$-intertwining linear embedding

$$
\left(\omega^{\square}\right)_{\mathrm{G}(V)} \triangleleft \mathrm{I}\left(s_{V, U}\right) .
$$

If $s_{U, V}>0$, then there exists a surjective $\mathrm{G}\left(U^{\square}\right)$-intertwining linear map

$$
\mathrm{I}\left(s_{U, V}\right) \rightarrow\left(\omega^{\square}\right)_{\mathrm{G}(V)} \triangle \subset I\left(s_{V, U}\right) .
$$

Proof. The first assertion is due to Rallis, see [R, Theorem II.1.1] and [MVW, Chapter 3, Theorem IV.7]. The second one is proved in [Ya, Theorems 1.3 and 1.4].

Write $q_{U}$ for the Witt index of $U$. Fix two sequences

$$
0=X_{0} \subset X_{1} \subset \cdots \subset X_{q_{U}} \quad \text { and } \quad X_{q_{U}}^{*} \supset \cdots \supset X_{1}^{*} \supset X_{0}^{*}=0
$$

of totally isotropic subspaces of $U$ such that for all $t=0,1, \cdots, q_{U}$,

$$
\left\{\begin{array}{l}
\operatorname{dim} X_{t}=\operatorname{dim} X_{t}^{*}=t ; \\
X_{t} \cap X_{t}^{*}=0 ; \text { and } \\
X_{t} \oplus X_{t}^{*} \text { is non-degenerate. }
\end{array}\right.
$$

Denote by $U_{t}$ the orthogonal complement of $X_{t} \oplus X_{t}^{*}$ in $U$. Write $\mathrm{P}\left(X_{t}\right)$ and $\mathrm{P}\left(X_{t}^{*}\right)$ for the parabolic subgroups of $\mathrm{G}(U)$ stabilizing $X_{t}$ and $X_{t}^{*}$, respectively. Then

$$
\mathrm{P}\left(X_{t}\right) \cap \mathrm{P}\left(X_{t}^{*}\right)=\mathrm{GL}\left(X_{t}\right) \times \mathrm{G}\left(U_{t}\right)
$$

is a common Levi factor of $\mathrm{P}\left(X_{t}\right)$ and $\mathrm{P}\left(X_{t}^{*}\right)$.

We need the following lemma (see [KR, Section 1]).

Lemma 2.3. Let $s \in \mathbb{C}$. As a representation of $\mathrm{G}(U) \times \mathrm{G}\left(U^{-}\right)$, $\mathrm{I}(s)$ possesses an equivariant filtration

$$
0=I_{-1}(s) \subset I_{0}(s) \subset I_{1}(s) \subset \cdots \subset I_{q_{U}}(s)=\mathrm{I}(s)
$$

with successive quotients

$$
R_{t}(s)=I_{t}(s) / I_{t-1}(s)=\operatorname{Ind}_{\mathrm{P}\left(X_{t}\right) \times \mathrm{P}\left(X_{t}\right)}^{\mathrm{G}(U) \times \mathrm{G}\left(U^{-}\right)}\left(\left(\chi_{V}\left|\operatorname{det}_{X_{t}}\right|_{\mathrm{F}}^{s+t} \otimes \chi_{V}\left|\operatorname{det}_{X_{t}}\right|_{\mathrm{F}}^{s+t}\right) \otimes C_{c}^{\infty}\left(\mathrm{G}\left(U_{t}\right)\right)\right),
$$

where $0 \leq t \leq q_{U}$, and

- $\operatorname{det}_{X_{t}}: \operatorname{GL}\left(X_{t}\right) \rightarrow \mathrm{F}^{\times}$denotes the reduced norm map, and $\chi_{V}$ is viewed as a character of $\mathrm{GL}\left(X_{t}\right)$ via the pullback through this map;

- $\mathrm{G}\left(U_{t}\right) \times \mathrm{G}\left(U_{t}\right)$ acts on $C_{c}^{\infty}\left(\mathrm{G}\left(U_{t}\right)\right)$ by left-right translation.

In particular, $R_{0}(s)=C_{c}^{\infty}(\mathrm{G}(U))$ is the regular representation. 
In view of Lemma 2.3, we make the following definition.

Definition: We say that an irreducible admissible smooth representation $\pi \otimes \sigma$ of $\mathrm{G}(U) \times \mathrm{G}\left(U^{-}\right)$lies on the boundary of $\mathrm{I}(\mathrm{s})$ if

$$
\operatorname{Hom}_{\mathrm{G}(U) \times \mathrm{G}\left(U^{-}\right)}\left(R_{t}(s), \pi \otimes \sigma\right) \neq 0 \quad \text { for some } 0<t \leq q_{U},
$$

where $R_{t}(s)$ is as in Lemma 2.3

Now we have:

Proposition 2.4. Proposition [2.1 holds when $\pi \otimes \sigma$ does not lie on the boundary of $\mathrm{I}\left(s_{U, V}\right)$.

Proof. Consider the doubling see-saw

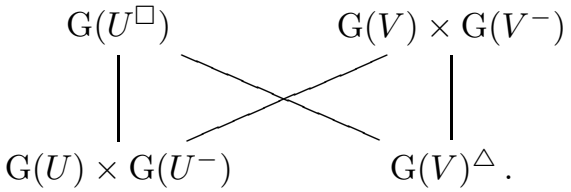

Given $\pi, \sigma \in \operatorname{Irr}(\mathrm{G}(U))$, the see-saw identity gives

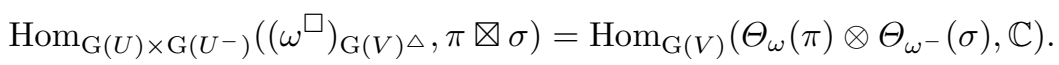

Assume that $s_{U, V}>0$ and $\pi \otimes \sigma$ does not lie on the boundary of $\mathrm{I}\left(s_{U, V}\right)$, then we have

$$
\begin{array}{rlr} 
& \operatorname{Hom}_{\mathrm{G}(V)}\left(\theta_{\omega}(\pi) \otimes \theta_{\omega^{-}}(\sigma), \mathbb{C}\right) \\
\hookrightarrow & \operatorname{Hom}_{\mathrm{G}(V)}\left(\Theta_{\omega}(\pi) \otimes \Theta_{\omega^{-}}(\sigma), \mathbb{C}\right) \\
= & \operatorname{Hom}_{\mathrm{G}(U) \times \mathrm{G}\left(U^{-}\right)}\left(\left(\omega^{\square}\right)_{\left.\mathrm{G}(V)^{\triangle}, \pi \otimes \sigma\right)}\right. \\
\hookrightarrow & \operatorname{Hom}_{\mathrm{G}(U) \times \mathrm{G}\left(U^{-}\right)}\left(\mathrm{I}\left(s_{U, V}\right), \pi \otimes \sigma\right) & \text { (by Lemma 2.2) } \\
\hookrightarrow & \operatorname{Hom}_{\mathrm{G}(U) \times \mathrm{G}\left(U^{-}\right)}\left(C_{c}^{\infty}(\mathrm{G}(U)), \pi \otimes \sigma\right) & \text { (by Lemma 2.3) } \\
\cong & \operatorname{Hom}_{\mathrm{G}(U)}(\pi \otimes \sigma, \mathbb{C}) .
\end{array}
$$

This proves the proposition.

\section{Some induced representations}

To complete the proof of Proposition 2.1, we need to consider representations $\pi \otimes \sigma$ of $\mathrm{G}(U) \times \mathrm{G}\left(U^{-}\right)$ which lie on the boundary of $\mathrm{I}\left(s_{U, V}\right)$. To deal with these, we study in this section some parabolically induced representations which will play an important role later on.

For smooth representations $\rho$ of $\mathrm{GL}\left(X_{t}\right)$ and $\sigma$ of $\mathrm{G}\left(U_{t}\right)\left(0 \leq t \leq q_{U}\right)$, we write

$$
\rho \rtimes \sigma:=\operatorname{Ind}_{\mathrm{P}\left(X_{t}\right)}^{\mathrm{G}(U)} \rho \otimes \sigma .
$$

More generally, the parabolic subgroup $P$ of $\mathrm{G}(U)$ stabilizing a flag

$$
0=X_{t_{0}} \subset X_{t_{1}} \subset X_{t_{2}} \subset \cdots \subset X_{t_{a}}
$$

has a Levi factor of the form $\operatorname{GL}\left(X_{t_{1}}\right) \times \mathrm{GL}\left(X_{t_{2}} / X_{t_{1}}\right) \times \cdots \times \mathrm{GL}\left(X_{t_{a}} / X_{t_{a-1}}\right) \times \mathrm{G}\left(U_{t_{a}}\right)$. We set

$$
\rho_{1} \times \cdots \times \rho_{a} \rtimes \sigma:=\operatorname{Ind}_{P}^{\mathrm{G}(U)} \rho_{1} \otimes \cdots \otimes \rho_{a} \otimes \sigma,
$$

where $\rho_{i}$ is a smooth representation of $\mathrm{GL}\left(X_{t_{i}} / X_{t_{i-1}}\right)$ and $\sigma$ is a smooth representation of $\mathrm{G}\left(U_{t_{a}}\right)$. Similarly, for the general linear group $\operatorname{GL}\left(X_{t_{a}}\right)$, we set

$$
\rho_{1} \times \cdots \times \rho_{a}:=\operatorname{Ind}_{Q}^{\mathrm{GL}\left(X_{t_{a}}\right)} \rho_{1} \otimes \cdots \otimes \rho_{a},
$$


where $Q$ is the parabolic subgroup of $\operatorname{GL}\left(X_{t_{a}}\right)$ stabilizing the flag (3.1). Respectively write $\mathrm{R}_{X_{t}}$ and $\mathrm{R}_{X_{t}^{*}}$ for the normalized Jacquet functors attached to $\mathrm{P}\left(X_{t}\right)$ and $\mathrm{P}\left(X_{t}^{*}\right)$.

Let $\eta: \mathrm{F}^{\times} \rightarrow \mathbb{C}^{\times}$be a character of $\mathrm{F}^{\times}$. Then $\eta^{\times a}\left(0 \leq a \leq q_{U}\right)$ is an irreducible representation of $\operatorname{GL}\left(X_{a}\right)$ (cf. Se] $)$. Here $\eta$ is viewed as a character of $\operatorname{GL}\left(X_{t} / X_{t-1}\right)(1 \leq t \leq a)$ via the pullback through the reduced norm map $\mathrm{GL}\left(X_{t} / X_{t-1}\right) \rightarrow \mathrm{F}^{\times}$. For every $\pi \in \operatorname{Irr}(\mathrm{G}(U))$, define

$$
\mathrm{m}_{\eta}(\pi):=\max \left\{0 \leq a \leq q_{U} \mid \pi \hookrightarrow \eta^{\times a} \rtimes \sigma \text { for some } \sigma \in \operatorname{Irr}\left(\mathrm{G}\left(U_{a}\right)\right)\right\} .
$$

Here $\pi \hookrightarrow \eta^{\times a} \rtimes \sigma$ means that there is an injective homomorphism from $\pi$ to $\eta^{\times a} \rtimes \sigma$ (similar notation will be used without further explanation).

The rest of this section is devoted to a proof of the following proposition.

Proposition 3.1. Assume that $\eta^{2}$ is non-trivial. Then for every $\pi \in \operatorname{Irr}(\mathrm{G}(U))$, there is a unique representation $\pi_{\eta} \in \operatorname{Irr}\left(\mathrm{G}\left(U_{a}\right)\right)$ such that $\pi \hookrightarrow \eta^{\times a} \rtimes \pi_{\eta}$, where $a:=\mathrm{m}_{\eta}(\pi)$. Moreover,

$$
\left\{\begin{array}{l}
\pi_{\eta} \cong\left(\mathrm{R}_{X_{a}}(\pi) \otimes\left(\eta^{-1}\right)^{\times a}\right)_{\mathrm{GL}\left(X_{a}\right)} \cong \operatorname{Hom}_{\mathrm{GL}\left(X_{a}\right)}\left(\left(\eta^{-1}\right)^{\times a}, \mathrm{R}_{X_{a}^{*}}(\pi)\right) ; \\
\pi \text { is isomorphic to the socle of } \eta^{\times a} \rtimes \pi_{\eta} ; \\
\mathrm{m}_{\eta}(\pi)=\mathrm{m}_{\eta}\left(\pi^{\vee}\right) ; \\
\left(\pi^{\vee}\right)_{\eta} \cong\left(\pi_{\eta}\right)^{\vee}
\end{array}\right.
$$

We begin with the following lemma.

Lemma 3.2. For all $\rho \in \operatorname{Irr}\left(\operatorname{GL}\left(X_{a}\right)\right)\left(0 \leq a \leq q_{U}\right)$ which is not isomorphic to $\eta^{\times a}$,

$$
\operatorname{Ext}_{\mathrm{GL}\left(X_{a}\right)}^{i}\left(\eta^{\times a}, \rho\right)=0 \quad(i \in \mathbb{Z}) .
$$

Proof. Since the Jacquet functor is exact and maps injective representations to injective representations, the second adjointness theorem of Bernstein implies that

$$
\operatorname{Ext}_{\mathrm{GL}\left(X_{a}\right)}^{i}\left(\eta^{\times a}, \rho\right) \cong \operatorname{Ext}_{\left(\mathrm{D}^{\times}\right)^{a}}^{i}\left(\eta^{\bigotimes a}, \overline{\mathrm{R}}(\rho)\right) .
$$

Here $\operatorname{GL}\left(X_{a}\right)$ is identified with $\mathrm{GL}_{a}(\mathrm{D})$ as usual, and $\overline{\mathrm{R}}$ denotes the normalized Jacquet functor attached to the minimal parabolic subgroup of $\mathrm{GL}_{a}(\mathrm{D})$ of lower triangular matrices. Note that $\rho \nsubseteq \eta^{\times a}$ implies that $\eta^{\bigotimes a}$ is not a subquotient of $\overline{\mathrm{R}}(\rho)$ (cf. [Be, Chapter 3, Section 2.1, Theorem 18]). Then it is easy to see that the right hand side of (3.4) vanishes.

By an easy homological algebra argument, Lemma 3.2 implies the following lemma.

Lemma 3.3. For all $\rho \in \operatorname{Irr}\left(\operatorname{GL}\left(X_{a}\right)\right)\left(0 \leq a \leq q_{U}\right)$ which is not isomorphic to $\eta^{\times a}$, and all $\sigma, \sigma^{\prime} \in \operatorname{Irr}\left(\mathrm{G}\left(U_{a}\right)\right)$,

$$
\operatorname{Ext}_{\mathrm{GL}\left(X_{a}\right) \times \mathrm{G}\left(U_{a}\right)}^{i}\left(\eta^{\times a} \otimes \sigma, \rho \otimes \sigma^{\prime}\right)=0 \quad(i \in \mathbb{Z}) .
$$

From now on, we assume that the character $\eta^{2} \neq 1$.

Lemma 3.4. Let $\sigma \in \operatorname{Irr}\left(\mathrm{G}\left(U_{a}\right)\right)\left(0 \leq a \leq q_{U}\right)$. Assume that $\mathrm{m}_{\eta}(\sigma)=0$ (as defined in (3.2)). Then

$$
\mathrm{R}_{X_{a}}\left(\eta^{\times a} \rtimes \sigma\right) \cong\left(\eta^{\times a} \otimes \sigma\right) \oplus \rho,
$$

where $\rho$ is a smooth representation of $\mathrm{GL}\left(X_{a}\right) \times \mathrm{G}\left(U_{a}\right)$ which has no irreducible subquotient of the form $\eta^{\times a} \otimes \sigma^{\prime}$ with $\sigma^{\prime} \in \operatorname{Irr}\left(\mathrm{G}\left(U_{a}\right)\right)$. Consequently, the socle of $\eta^{\times a} \rtimes \sigma$ is irreducible.

Proof. Denote by $\rho$ the kernel of the natural surjective homomorphism

$$
\mathrm{R}_{X_{a}}\left(\eta^{\times a} \rtimes \sigma\right) \rightarrow \eta^{\times a} \otimes \sigma .
$$

As in the proof of GT, Lemma 5.2], using an explication of the Geometric Lemma of BernsteinZelevinsky ( $c f$. T, Lemma 5.1] and [Ha]), the assumption of the lemma implies that $\rho$ contains no 
irreducible subquotient of the form $\eta^{\times a} \otimes \sigma^{\prime}$ with $\sigma^{\prime} \in \operatorname{Irr}\left(\mathrm{G}\left(U_{a}\right)\right)$. Then Lemma 3.3 implies that the surjective homomorphism (3.5) splits. This proves the first assertion of the lemma. The second assertion then easily follows as in [GT, Lemma 5.2].

In the rest of this section, let $\pi \in \operatorname{Irr}(\mathrm{G}(U))$ and put $a:=\mathrm{m}_{\eta}(\pi)$. Then there is an irreducible representation $\sigma \in \operatorname{Irr}\left(\mathrm{G}\left(U_{a}\right)\right)$ such that $\pi \hookrightarrow \eta^{\times a} \rtimes \sigma$. Induction-by-steps shows that $\mathrm{m}_{\eta}(\sigma)=0$.

Lemma 3.5. One has that

$$
\mathrm{R}_{X_{a}}(\pi) \cong\left(\eta^{\times a} \otimes \sigma\right) \oplus \rho,
$$

where $\rho$ is a smooth representation of $\mathrm{GL}\left(X_{a}\right) \times \mathrm{G}\left(U_{a}\right)$ which has no irreducible subquotient of the form $\eta^{\times a} \otimes \sigma^{\prime}$ with $\sigma^{\prime} \in \operatorname{Irr}\left(\mathrm{G}\left(U_{a}\right)\right)$.

Proof. Since $\mathrm{R}_{X_{a}}(\pi)$ is a subrepresentation of $\mathrm{R}_{X_{a}}\left(\eta^{\times a} \rtimes \sigma\right)$ and has $\eta^{\times a} \bowtie \sigma$ as an irreducible quotient, the lemma easily follows from Lemma 3.4

Lemma 3.6. One has that

$$
\mathrm{R}_{X_{a}^{*}}(\pi) \cong\left(\left(\eta^{-1}\right)^{\times a} \otimes \sigma\right) \oplus \rho,
$$

where $\rho$ is a smooth representation of $\mathrm{GL}\left(X_{a}\right) \times \mathrm{G}\left(U_{a}\right)$ which has no irreducible subquotient of the form $\left(\eta^{-1}\right)^{\times a} \otimes \sigma^{\prime}$ with $\sigma^{\prime} \in \operatorname{Irr}\left(\mathrm{G}\left(U_{a}\right)\right)$.

Proof. Note that $\mathrm{P}\left(X_{a}\right)$ is conjugate to $\mathrm{P}\left(X_{a}^{*}\right)$ by an element $w \in \mathrm{G}(U)$ such that $w$ is the identity on $U_{a}$ and $w$ exchanges $X_{a}$ and $X_{a}^{*}$. Via conjugation by $w$, we see that Lemma 3.6 is equivalent to Lemma 3.5 .

Lemma 3.5 and Lemma 3.6 imply that

$$
\sigma \cong\left(\mathrm{R}_{X_{a}}(\pi) \otimes\left(\eta^{-1}\right)^{\times a}\right)_{\mathrm{GL}\left(X_{a}\right)} \cong \operatorname{Hom}_{\mathrm{GL}\left(X_{a}\right)}\left(\left(\eta^{-1}\right)^{\times a}, \mathrm{R}_{X_{a}^{*}}(\pi)\right) .
$$

This proves the uniqueness assertion of Proposition 3.1, as well as the first assertion of (3.3). The second assertion of (3.3) is then implied by the last assertion of Lemma 3.4.

Lemma 3.7. One has that

$$
\pi^{\vee} \hookrightarrow \eta^{\times a} \rtimes \sigma^{\vee}
$$

Proof. Lemma 3.6 implies that

$$
\left(\eta^{-1}\right)^{\times a} \otimes \sigma \hookrightarrow \mathrm{R}_{X_{a}^{*}}(\pi) .
$$

By dualizing and using the second adjointness theorem, we see that

$$
\mathrm{R}_{X_{a}}\left(\pi^{\vee}\right) \rightarrow \eta^{\times a} \otimes \sigma^{\vee} .
$$

This implies that $\pi^{\vee} \hookrightarrow \eta^{\times a} \rtimes \sigma^{\vee}$.

Lemma 3.7 implies that $\mathrm{m}_{\eta}\left(\pi^{\vee}\right) \geq \mathrm{m}_{\eta}(\pi)$. The same argument shows that $\mathrm{m}_{\eta}(\pi) \geq \mathrm{m}_{\eta}\left(\pi^{\vee}\right)$. This proves that $\mathrm{m}_{\eta}\left(\pi^{\vee}\right)=\mathrm{m}_{\eta}(\pi)$. Lemma 3.7 then further implies that $\left(\pi^{\vee}\right)_{\eta} \cong\left(\pi_{\eta}\right)^{\vee}$. This finally finishes the proof of Proposition 3.1. 


\section{Induced representations and theta correspondence}

In this section, we apply the results of the previous section to the theta correspondence. Write $\eta^{\prime}: \mathrm{F}^{\times} \rightarrow \mathbb{C}^{\times}$for the character such that

$$
\eta^{\prime} \cdot \chi_{V}=\eta \cdot \chi_{U}
$$

Then $\left(\eta^{\prime}\right)^{2} \neq 1$ since $\eta^{2} \neq 1$. Denote by $q_{V}$ the Witt index of $V$. Similarly to (2.5), we fix two sequences

$$
0=Y_{0} \subset Y_{1} \subset \cdots \subset Y_{q_{V}} \quad \text { and } \quad Y_{q_{V}}^{*} \supset \cdots \supset Y_{1}^{*} \supset Y_{0}^{*}=0
$$

of totally isotropic subspaces of $V$ with the analogous properties as in (2.5). We apply the analogous notation as in the last section to the space $V$. In particular, $\mathrm{m}_{\eta^{\prime}}\left(\pi^{\prime}\right)$ is defined for every $\pi^{\prime} \in \operatorname{Irr}(\mathrm{G}(V))$. Define $\pi_{\eta}\left(\pi \in \operatorname{Irr}(\mathrm{G}(U))\right.$ and $\pi_{\eta^{\prime}}^{\prime}$ as in Proposition 3.1 .

For all integers $0 \leq a \leq q_{U}$ and $0 \leq k \leq q_{V}$, write $\omega_{a, k}:=\omega_{U_{a}, V_{k}, \psi}$, which is an irreducible smooth representation of $\left(\mathrm{G}\left(U_{a}\right) \times \mathrm{G}\left(V_{k}\right)\right) \ltimes \mathrm{H}\left(U_{a} \otimes_{\mathrm{D}} V_{k}\right)$, as defined in Section 2.

The rest of this section is devoted to a proof of the following key proposition.

Proposition 4.1. Assume that

$$
\eta \neq \chi_{V}|\cdot|_{\mathrm{F}}^{s_{V, U}+1} \quad \text { and } \quad \eta^{\prime} \neq \chi_{U}|\cdot|_{\mathrm{F}}^{s_{U, V}+1} .
$$

Then for all $\pi \in \operatorname{Irr}(\mathrm{G}(U))$ and $\pi^{\prime} \in \operatorname{Irr}(\mathrm{G}(V))$ such that $\operatorname{Hom}_{\mathrm{G}(U) \times \mathrm{G}(V)}\left(\omega, \pi \bigotimes \pi^{\prime}\right) \neq 0$, one has

$$
\mathrm{m}_{\eta}(\pi)=\mathrm{m}_{\eta^{\prime}}\left(\pi^{\prime}\right),
$$

and there is a linear embedding

$$
\operatorname{Hom}_{\mathrm{G}(U) \times \mathrm{G}(V)}\left(\omega, \pi \bigotimes \pi^{\prime}\right) \hookrightarrow \operatorname{Hom}_{\mathrm{G}\left(U_{a}\right) \times \mathrm{G}\left(V_{a}\right)}\left(\omega_{a, a}, \pi_{\eta} \otimes \pi_{\eta^{\prime}}^{\prime}\right),
$$

where $a:=\mathrm{m}_{\eta}(\pi)$.

For each right D-vector space $X$, write $X^{\iota}$ for the left D-vector which equals $X$ as an abelian group and whose scalar multiplication is given by

$$
\alpha v:=v \alpha^{\iota}, \quad \alpha \in \mathrm{D}, v \in X^{\iota} .
$$

We first recall the well-known computation of the Jacquet module of the Weil representation (see $\underline{K}$, Theorem 2.8] and [MVW, Chapter 3, Section IV.5]).

Lemma 4.2. For each $0 \leq a \leq q_{U}$, the normalized Jacquet module $\mathrm{R}_{X_{a}}(\omega)$ has a $\mathrm{GL}\left(X_{a}\right) \times \mathrm{G}\left(U_{a}\right) \times$ $\mathrm{G}(V)$-equivariant filtration

$$
\mathrm{R}_{X_{a}}(\omega)=R_{0} \supset R_{1} \supset \cdots \supset R_{a^{\prime}} \supset R_{a^{\prime}+1}=0
$$

whose successive quotient is

$J_{k}:=R_{k} / R_{k+1} \cong \operatorname{Ind}_{\mathrm{P}\left(X_{a-k}, X_{a}\right) \times \mathrm{G}\left(U_{a}\right) \times \mathrm{P}\left(Y_{k}\right)}^{\mathrm{GL}\left(X_{a}\right) \times \mathrm{G}\left(U_{a}\right) \times \mathrm{G}(V)}\left(\chi_{V}\left|\operatorname{det}_{X_{a-k}}\right|_{\mathrm{F}}^{s_{V, U}+a-k} \otimes C_{c}^{\infty}\left(\operatorname{Isom}\left(X_{a}^{\iota} / X_{a-k}^{\iota}, Y_{k}\right)\right) \otimes \omega_{a, k}\right)$, where

- $a^{\prime}:=\min \left\{a, q_{V}\right\}$ and $0 \leq k \leq a^{\prime}$

- $\mathrm{P}\left(X_{a-k}, X_{a}\right)$ is the parabolic subgroup of $\mathrm{GL}\left(X_{a}\right)$ stabilizing $X_{a-k}$;

- $\operatorname{det}_{X_{a-k}}: \mathrm{GL}\left(X_{a-k}\right) \rightarrow \mathrm{F}^{\times}$denotes the reduced norm map, and $\chi_{V}$ is viewed as a character of $\mathrm{GL}\left(X_{a-k}\right)$ via the pullback through this map;

- $\operatorname{Isom}\left(X_{a}^{\iota} / X_{a-k}^{\iota}, Y_{k}\right)$ is the set of D-linear isomorphisms from $X_{a}^{\iota} / X_{a-k}^{\iota}$ to $Y_{k}$, and $\mathrm{GL}\left(X_{a} / X_{a-k}\right) \times$ $\mathrm{GL}\left(Y_{k}\right)$ acts on $C_{c}^{\infty}\left(\operatorname{Isom}\left(X_{a}^{\iota} / X_{a-k}^{\iota}, Y_{k}\right)\right)$ as

$$
((b, c) \cdot f)(g)=\chi_{V}(\operatorname{det} b) \chi_{U}(\operatorname{det} c) f\left(c^{-1} g b\right),
$$

for $(b, c) \in \mathrm{GL}\left(X_{a} / X_{a-k}\right) \times \mathrm{GL}\left(Y_{k}\right), f \in C_{c}^{\infty}\left(\operatorname{Isom}\left(X_{a}^{\iota} / X_{a-k}^{\iota}, Y_{k}\right)\right)$ and $g \in \operatorname{Isom}\left(X_{a}^{\iota} / X_{a-k}^{\iota}, Y_{k}\right)$. 
In particular, if $a^{\prime}=a$, then the bottom piece of the filtration is

$$
J_{a} \cong \operatorname{Ind}_{\mathrm{GL}\left(X_{a}\right) \times \mathrm{G}\left(U_{a}\right) \times \mathrm{P}\left(Y_{a}\right)}^{\mathrm{GL}\left(X_{a}\right) \times G\left(U_{a}\right) \times \mathrm{G}(V)}\left(C_{c}^{\infty}\left(\operatorname{Isom}\left(X_{a}^{\iota}, Y_{a}\right)\right) \otimes \omega_{a, a}\right) .
$$

The following lemma is an observation of [GT].

Lemma 4.3. Let $a, k$ and $J_{k}$ be as in Lemma 4.2. Assume that $\eta \neq \chi_{V}|\cdot|_{\mathrm{F}}^{s_{V, U}+1}$. Then for all $\sigma \in \operatorname{Irr}\left(\mathrm{G}\left(U_{a}\right)\right)$ and $\pi^{\prime} \in \operatorname{Irr}(\mathrm{G}(V))$,

$$
\operatorname{Hom}_{\mathrm{GL}\left(X_{a}\right) \times \mathrm{G}\left(U_{a}\right) \times \mathrm{G}(V)}\left(J_{k}, \eta^{\times a} \otimes \sigma \otimes \pi^{\prime}\right)=0
$$

whenever $k \neq a$.

Proof. Using the second adjointness theorem, it suffices to show that

$$
\operatorname{Hom}_{\mathrm{GL}\left(X_{a-k}\right)}\left(\chi_{V}\left|\operatorname{det}_{X_{a-k}}\right|_{\mathrm{F}}^{S_{V, U}+a-k}, \overline{\mathrm{R}}_{X_{a-k}, X_{a}}\left(\eta^{\times a}\right)\right)=0,
$$

where $\overline{\mathrm{R}}_{X_{a-k}, X_{a}}$ denotes the normalized Jacquet functor attached to the parabolic subgroup of $\mathrm{GL}\left(X_{a}\right)$ stabilizing a complement of $X_{a-k}$ in $X_{a}$. By analysing the cuspidal data, we know that every irreducible subrepresentation of $\overline{\mathrm{R}}_{X_{a-k}, X_{a}}\left(\eta^{\times a}\right)$ is isomorphic to $\eta^{\times(a-k)} \otimes \eta^{\times k}$, as a representation of $\mathrm{GL}\left(X_{a-k}\right) \times \mathrm{GL}\left(X_{a} / X_{a-k}\right)$. Therefore the lemma follows.

Now we come to the proof of Proposition 4.1 Put $a:=\mathrm{m}_{\eta}(\pi)$. Then we have

$$
\begin{aligned}
& 0 \neq \operatorname{Hom}_{\mathrm{G}(U) \times \mathrm{G}(V)}\left(\omega, \pi \otimes \pi^{\prime}\right) \\
& \hookrightarrow \operatorname{Hom}_{\mathrm{G}(U) \times \mathrm{G}(V)}\left(\omega,\left(\eta^{\times a} \rtimes \pi_{\eta}\right) \otimes \pi^{\prime}\right) \\
& =\operatorname{Hom}_{\mathrm{GL}\left(X_{a}\right) \times \mathrm{G}\left(U_{a}\right) \times \mathrm{G}(V)}\left(\mathrm{R}_{X_{a}}(\omega), \eta^{\times a} \otimes \pi_{\eta} \otimes \pi^{\prime}\right) \\
& \hookrightarrow \operatorname{Hom}_{\mathrm{GL}\left(X_{a}\right) \times \mathrm{G}\left(U_{a}\right) \times \mathrm{G}(V)}\left(J_{a}, \eta^{\times a} \otimes \pi_{\eta} \otimes \pi^{\prime}\right) \quad \text { (by Lemma 4.3) } \\
& \cong \operatorname{Hom}_{\mathrm{GL}\left(X_{a}\right) \times \mathrm{G}\left(U_{a}\right) \times \mathrm{GL}\left(Y_{a}\right) \times \mathrm{G}\left(V_{a}\right)}\left(C_{c}^{\infty}\left(\operatorname{Isom}\left(X_{a}^{\iota}, Y_{a}\right)\right) \otimes \omega_{a, a}, \eta^{\times a} \otimes \pi_{\eta} \otimes \mathrm{R}_{Y_{a}^{*}}\left(\pi^{\prime}\right)\right)
\end{aligned}
$$

(by the second ajointness theorem)

$\cong \operatorname{Hom}_{\mathrm{G}\left(U_{a}\right) \times \mathrm{GL}\left(Y_{a}\right) \times \mathrm{G}\left(V_{a}\right)}\left(\left(\eta^{\prime-1}\right)^{\times a} \otimes \omega_{a, a}, \pi_{\eta} \otimes \mathrm{R}_{Y_{a}^{*}}\left(\pi^{\prime}\right)\right)$

$\cong \operatorname{Hom}_{\mathrm{G}\left(U_{a}\right) \times \mathrm{G}\left(V_{a}\right)}\left(\omega_{a, a}, \pi_{\eta} \otimes \pi_{a}^{\prime}\right)$,

where

$$
\pi_{a}^{\prime}:=\operatorname{Hom}_{\mathrm{GL}\left(Y_{a}\right)}\left(\left(\eta^{\prime-1}\right)^{\times a}, \mathrm{R}_{Y_{a}^{*}}\left(\pi^{\prime}\right)\right) .
$$

Therefore $\pi_{a}^{\prime} \neq 0$, and hence

$$
\operatorname{Hom}_{\mathrm{GL}\left(Y_{a}\right) \times \mathrm{G}\left(V_{a}\right)}\left(\left(\eta^{\prime-1}\right)^{\times a} \otimes \pi_{a}^{\prime}, \mathrm{R}_{Y_{a}^{*}}\left(\pi^{\prime}\right)\right) \neq 0 .
$$

Dualizing and using the second adjointness theorem, we see that

$$
\operatorname{Hom}_{\mathrm{GL}\left(Y_{a}\right) \times \mathrm{G}\left(V_{a}\right)}\left(\mathrm{R}_{Y_{a}}\left(\pi^{\prime \vee}\right), \eta^{\prime \times a} \otimes \pi_{a}^{\prime \vee}\right) \neq 0 .
$$

This proves that

$$
\mathrm{m}_{\eta^{\prime}}\left(\pi^{\prime}\right)=\mathrm{m}_{\eta^{\prime}}\left(\pi^{\prime \vee}\right) \geq a=\mathrm{m}_{\eta}(\pi) .
$$

The same argument shows that $\mathrm{m}_{\eta}(\pi) \geq \mathrm{m}_{\eta^{\prime}}\left(\pi^{\prime}\right)$, and hence $\mathrm{m}_{\eta^{\prime}}\left(\pi^{\prime}\right)=\mathrm{m}_{\eta}(\pi)$. Therefore $\pi_{a}^{\prime} \cong \pi_{\eta^{\prime}}^{\prime}$ by Proposition 3.1. This finishes the proof of Proposition 4.1 . 


\section{Proof of Proposition 2.1}

In this section, we finish the proof of Proposition 2.1 by induction on $\operatorname{dim} U$. As in Proposition 2.1, let $\pi, \sigma \in \operatorname{Irr}(\mathrm{G}(U))$ and assume that $s_{U, V}>0$. In view of Proposition 2.4 we may assume that $\pi \otimes \sigma$ lies on the boundary of $\mathrm{I}\left(s_{U, V}\right)$. Then there is an integer $0<t \leq q_{U}$ such that

$$
\operatorname{Hom}_{\mathrm{G}(U) \times \mathrm{G}\left(U^{-}\right)}\left(R_{t}\left(s_{U, V}\right), \pi \otimes \sigma\right) \neq 0 .
$$

Note that

$$
=\sum_{\pi^{\prime} \in \operatorname{Irr}(\mathrm{G}(V))} \operatorname{dim} \operatorname{Hom}_{\mathrm{G}(U) \times \mathrm{G}(V)}\left(\omega, \pi \otimes \pi^{\prime}\right) \cdot \operatorname{dim} \operatorname{Hom}_{\mathrm{G}(U) \times \mathrm{G}(V)}\left(\omega^{-}, \sigma \otimes \pi^{\prime \vee}\right) .
$$

We assume that the value of the above equality is non-zero, as Proposition 2.1 is otherwise trivial. Then there is an irreducible representation $\pi^{\prime} \in \operatorname{Irr}(\mathrm{G}(V))$ such that

$$
\operatorname{Hom}_{\mathrm{G}(U) \times \mathrm{G}(V)}\left(\omega, \pi \otimes \pi^{\prime}\right) \neq 0 \quad \text { and } \quad \operatorname{Hom}_{\mathrm{G}(U) \times \mathrm{G}(V)}\left(\omega^{-}, \sigma \otimes \pi^{\prime \vee}\right) \neq 0 .
$$

By the second adjointness theorem, (5.1) implies that

$$
\operatorname{Hom}_{\mathrm{GL}\left(X_{t}\right)}\left(\chi\left|\operatorname{det}_{X_{t}}\right|_{\mathrm{F}}^{s_{U, V}+t}, \mathrm{R}_{X_{t}^{*}}(\pi)\right) \neq 0 .
$$

Put

$$
\eta:=\chi_{V}|\cdot|^{s_{V, U}-2 t+1} \text { and } \quad \eta^{\prime}:=\chi_{U}|\cdot|^{s_{V, U}-2 t+1} .
$$

Using the second adjointness theorem and the Langlands parameter of the character $\chi_{V}\left|\operatorname{det}_{X_{t}}\right|_{\mathrm{F}}^{s_{V, U}-t}$, (5.4) implies that

$$
\mathrm{m}_{\eta}(\pi)=\mathrm{m}_{\eta}\left(\pi^{\vee}\right)>0
$$

Noting that

$$
\eta \neq \chi_{V}|\cdot|_{\mathrm{F}}^{s_{V, U}+1} \quad \text { and } \quad \eta^{\prime} \neq \chi_{U}|\cdot|_{\mathrm{F}}^{s_{U, V}+1},
$$

Proposition 4.1 (and its analog for $\omega^{-}$) then implies that

$$
\mathrm{m}_{\eta}(\pi)=\mathrm{m}_{\eta^{\prime}}\left(\pi^{\prime}\right)=\mathrm{m}_{\eta^{\prime}}\left(\pi^{\prime \vee}\right)=\mathrm{m}_{\eta}(\sigma) .
$$

By the induction assumption, Proposition 2.1 holds for the pair $\left(U_{a}, V_{a}\right)$, where $a:=\mathrm{m}_{\eta}(\pi)$. As we have seen at the end of the introduction, this implies that Theorem 1.2 holds for the pair $\left(\mathrm{G}\left(U_{a}\right), \mathrm{G}\left(V_{a}\right)\right)$. Together with Proposition 4.1, this implies that

$$
\pi_{\eta^{\prime}}^{\prime} \cong \theta_{\omega_{a, a}}\left(\pi_{\eta}\right)
$$

and

$$
\pi_{\eta} \cong \theta_{\omega_{a, a}}\left(\pi_{\eta^{\prime}}^{\prime}\right) \quad \text { and } \quad \sigma_{\eta} \cong \theta_{\omega_{a, a}^{-}}\left(\left(\pi_{\eta^{\prime}}^{\prime}\right)^{\vee}\right) .
$$

Here $\omega_{a, a}^{-}:=\omega_{U_{a}, V_{a}, \psi}^{-}$. Proposition 4.1 and (5.5) imply that $\pi^{\prime}$ is isomorphic to the socle of $\eta^{\prime \times a} \rtimes$ $\theta_{\omega_{a, a}}\left(\pi_{\eta}\right)$. Therefore, there is a unique $\pi^{\prime} \in \operatorname{Irr}(\mathrm{G}(U))$ which satisfies (5.3). Then Proposition 4.1 implies that the value of (5.2) is 1 .

On the other hand, (5.6) and the induction assumption imply that $\pi_{\eta}^{\vee} \cong \sigma_{\eta}$, which further implies that $\pi^{\vee} \cong \sigma$ by Proposition 3.1. Therefore (2.3) of Proposition 2.1] is an equality. This finishes the proof of Proposition 2.1 


\section{REFERENCES}

[Be] J. Bernstein, Draft of: representations of p-adic groups, Lectures by Joseph Bernstein, Harvard University Fall 1992, written by Karl E. Rumelhart.

[GT] W. T. Gan and S. Takeda, A proof of the Howe duality conjecture, to appear in Journal of AMS.

[H1] R. Howe, $\theta$-series and invariant theory, in Automorphic Forms, Representations and L-functions, Proceedings of Symposia in Pure Mathematics Vol. 33 (1979), part 1, pp. 275-285.

[H2] R. Howe, Transcending classical invariant theory, J. Amer. Math. Soc. 2 (1989), 535-552.

[Ha] M. Hanzer, Inducirane reprezentacije hermitskih kvaternionskih grupa, Ph.D. thesis, University of Zagreb (2005).

[K] S. S. Kudla, On the local theta-correspondence, Invent. Math. 83 (1986), 229-255.

$[\mathrm{KR}]$ S. S. Kudla and S. Rallis, On first occurrence in the local theta correspondence, Automorphic representations, $L$-functions and applications: progress and prospects, Ohio State Univ. Math. Res. Inst. Publ. 11, de Gruyter, Berlin, 2005, pp. 273-308.

[LSTi] J.-S. Li, B. Sun and Y. Tian, The multiplicity one conjecture for local theta correspondences, Invent. Math., 184 (2011), 117-124.

[LSTa] Y. Lin, B. Sun and S. Tan, MVW-extensions of real quaternionic classical groups, Math. Z. 277 (2014), no. 1-2, 81-89.

[M] A. Minguez, Correspondance de Howe explicite: paires duales de type II, Ann. Sci. Éc. Norm. Supér. 41 (2008), $717-741$.

[MVW] C. Møglin, M.-F. Vignéras, and J.-L. Waldspurger, Correspondances de Howe sur un corps p-adique, Lecture Notes in Mathematics 1291, Springer-Verlag, Berlin, 1987.

[R] S. Rallis, On the Howe duality conjecture, Compositio Math. 51, (1984), 333-399.

[Se] V. Sécherre, Proof of the Tadić conjecture (U0) on the unitary dual of $\mathrm{GL}_{m}$ (D), J. Reine Angew.Math. 626 (2009), 187-203.

[S] B. Sun, Dual pairs and contragredients of irreducible representations, Pacific. J. Math., 249, (2011), $485-494$.

[SZ] B. Sun and C.-B. Zhu, Conservation relations for local theta correspondence, to appear in Journal of AMS. arXiv:1204.2969.

[T] M. Tadić, Structure arising from induction and Jacquet modules of representations of classical p-adic groups, Journal of Algebra vol. 177 (1995), Pg. 1-33.

[Wa] J.-L. Waldspurger, Démonstration d'une conjecture de dualité de Howe dans le cas $p$-adique, $p \neq 2$, Festschrift in honor of I. I. Piatetski-Shapiro on the occasion of his sixtieth birthday, Part I, Israel Math. Conf. Proc. 2, Weizmann, Jerusalem, 1990, pp. 267-324.

[We] A. Weil, Sur certaines d'opérateurs unitaires, Acta Math. 111 (1964), 143-211.

[Ya] S. Yamana, Degenerate principal series representations for quaternionic unitary groups, Israel J. Math. 185, (2011), $77-124$.

Department of Mathematics, National University of Singapore, 10 Lower Kent Ridge Road, Singapore 119076

E-mail address: matgwt@nus.edu.sg

Hua Loo-Keng Key Laboratory of Mathematics, Institute of Mathematics, Academy of Mathematics and Systems Science, CAS, Beijing, 100190, P.R. China

E-mail address: sun@math.ac.cn 